\title{
HOMOMORPHIC IMAGES OF FINITE SUBDIRECTLY IRREDUCIBLE UNARY ALGEBRAS
}

\author{
J. JEŽEK, P. MARKOVIĆ AND D. STANOVSKÝ
}

\begin{abstract}
We prove that a finite unary algebra with at least two operation symbols is a homomorphic image of a (finite) subdirectly irreducible algebra if and only if the intersection of all its subalgebras which have at least two elements is nonempty.
\end{abstract}

We are concerned with the following natural question: Which algebras are homomorphic images of subdirectly irreducible algebras?

A necessary condition, discovered in [3], [4] and [5], for an algebra $A$ with at least one at least binary operation to be a homomorphic image of some subdirectly algebra, is that the intersection of all ideals of $A$ is nonempty. (By an ideal of $A$ we mean a nonempty subset $I$ such that $f\left(a_{1}, \ldots, a_{n}\right) \in I$ whenever $f$ is a fundamental operation and $a_{1}, \ldots, a_{n}$ are elements of $A$ with $a_{i} \in I$ for at least one $i$.) It was proved in [2] and independently in [8] that the condition is also sufficient. In fact, it was proved in those two papers that any algebra $A$ with at least one at least binary operation and with a smallest ideal is isomorphic to a factor of a subdirectly irreducible algebra $B$ through its monolith, and the construction is such that if $A$ is finite then also $B$ is finite. The case that remains is that of algebras with only unary operations. (Note that nullary operations play no role in investigation of congruences.)

If there is just one unary operation, the characterization is simple; see e.g. [9] or [2]. For two or more unary operations, the situation is more complicated. In the present paper we are going to characterize finite algebras with at least two unary operations that are homomorphic images of a subdirectly irreducible algebra. We leave it as an open problem to do the same for infinite algebras. An example found in [2] suggests that it will be probably much harder to characterize such unary algebras that are isomorphic to a subdirectly irreducible algebra through its monolith.

1991 Mathematics Subject Classification. 08A60, 08B26.

Key words and phrases. subdirectly irreducible unary algebra.

While working on this paper the first and the third authors were partially supported by the Grant Agency of the Czech Republic, grant \#201/02/0594 and by the institutional grant MSM113200007; the second author was supported by the Ministry of Science, Technologies and Development of Republic of Serbia, grant no. 1227. 
One can ask similar questions in particular varieties of algebras. For instance, the construction from [8] yields for an idempotent algebra an idempotent subdirectly irreducible one. (In fact, it preserves any identity of the form $t(x) \approx x$, where $t$ is a unary term. And if the algebra contains no proper ideal, then the construction preserves any identity in one variable.) However, it seems that no other interesting identities are kept.

Indeed, in many varieties almost no algebras can be represented as homomorphic images of subdirectly irreducibles - for instance in distributive lattices, for an obvious reason. On the other hand, in several familiar varieties the answer is known to be similar to the general case. It is proved in [1] that a semigroup is a homomorphic image of some subdirectly irreducible semigroup if and only if the intersection of all its ideals is nonempty. However, there are finite semigroups (e.g. any right zero band) which are not isomorphic to the factor of a finite subdirectly irreducible semigroup over its monolith. Every quasigroup, group and every lattice (indeed, all of them have no non-trivial ideals, in our sense) is also a homomorphic image of a subdirectly irreducible one (quasigroup, group, lattice) and for a finite one the s.i. can be constructed finite. For lattices, an easy construction was found by Ralph Freese (unpublished). For quasigroups and groups, a solution was found by R. McKenzie and D. Stanovský [7].

Throughout this paper let $\sigma$ be a finite set of unary operation symbols, $|\sigma| \geq 2$. By a unary algebra we mean a $\sigma$-algebra. By a word we mean a word over the alphabet $\sigma$.

For a word $w=f_{1} \ldots f_{n}$ and any $i=0, \ldots, n-1$ we define a word $w^{[i]}=f_{i+1} \ldots f_{n} f_{1} \ldots f_{i}$. This is a word of the same length as $w$. We put $w^{i}=w w \ldots w$ where $w$ is repeated $i$ times.

Let $A$ be a unary algebra; let $a \in A$ and $w=f_{1} \ldots f_{n}$ be a word. For $i=0, \ldots, n$ we put $w_{\langle i\rangle}(a)=f_{i} \ldots f_{2} f_{1}(a)$ (so that $\left.w_{\langle 0\rangle}(a)=a\right)$. By an e-pair for $A$ we mean a pair $a, w$ where $a \in A, w$ is a word of some positive length $n, w_{\langle n\rangle}(a)=a$ and $A=\left\{w_{\langle 0\rangle}(a), \ldots, w_{\langle n-1\rangle}(a)\right\}$. (Informally, an e-pair is a path through all elements of $A$ using the operations.) An e-pair $a, w$ is said to start at $a$; $w$ is called the e-pair's path; the e-pair's length is the length of its path (clearly, the length is at least $|A|$ ).

It is easy to see that a finite unary algebra $A$ has an e-pair if and only if it has no proper subalgebra; and if $a, w$ is an e-pair for $A$ then $w_{\langle i\rangle}(a), w^{[i]}$ is an e-pair for $A$ for any $i=0, \ldots, n-1$, where $n$ is the length of $w$. Consequently, if $A$ has no proper subalgebra then it has an e-pair starting at any of its elements.

An e-pair $a, w$ for $A$ is said to be an h-pair if $|A|$ equals the length of $w$.

Lemma 1. Let $a, w$ be an e-pair for a finite unary algebra $A$. Then there exist a finite unary algebra $B$ and an element $b \in B$ such that $A$ is a homomorphic image of $B$ and $b, w$ is an h-pair for $B$. 
Proof. Put $w=f_{1} \ldots f_{n}$. For $i=0, \ldots, n-1$ put $a_{i}=w_{\langle i\rangle}(a)$. For every $c \in A$ denote by $p_{c}$ the number of occurrences of $c$ in $w_{\langle 0\rangle}(a), \ldots, w_{\langle n-1\rangle}(a)$. Put $B=\left\{\left(a_{i}, j\right): 0 \leq i<n, 0 \leq j<p_{a_{i}}\right\}$. For $\left(a_{i}, j\right) \in B$ and $f \in \sigma$ put $f\left(a_{i}, j\right)=\left(f\left(a_{i}\right), k\right)$ where $k=\left|\left\{m: 0 \leq m<i, f\left(a_{i}\right)=a_{m}\right\}\right|$. Then $\left(a_{i}, j\right) \mapsto a_{i}$ is a homomorphism of $B$ onto $A$ and $(a, 0), w$ is an h-pair for $B$.

A word $w=f_{1} \ldots f_{n}$ is said to be irreducible if $w \neq w^{[i]}$ for all $1 \leq i<n$. It is easy to see that a word is irreducible if and only if it cannot be expressed as $u^{j}$ for a word $u$ and an integer $j \geq 2$. An e-pair is said to be irreducible if its path is irreducible.

Lemma 2. Let $a, w$ be an e-pair for a finite unary algebra $A$. Then there exists a finite unary algebra $B$ such that $A$ is a homomorphic image of $B$ and $B$ has an irreducible h-pair.

Proof. Let $w=f_{1} \ldots f_{n}$ for some $f_{i} \in \sigma$. Take a symbol $g \in \sigma$ different from $f_{1}$ and put $b=f_{1} g^{2 n}(a)$. Let $i$ be such that $b=w_{\langle i\rangle}(a)$ and put $h_{1}=f_{i+1}, h_{2}=f_{i+2}, \ldots, h_{n}=f_{i}, \ldots, h_{m}=f_{n}$, so that $a=h_{m} \ldots h_{1}(b)$, $A=\left\{b, h_{1}(b), \ldots, h_{m} \ldots h_{1}(b)\right\}$ and $m<2 n$. Thus $a, g^{2 n} f_{1} h_{1} \ldots h_{m}$ is an irreducible e-pair for $A$. Now use Lemma 1.

Lemma 3. Let $a, w$ be an irreducible h-pair for a finite unary algebra $A$. Then $A$ is a homomorphic image of a finite subdirectly irreducible unary algebra. Moreover, $A$ is isomorphic to a factor of a finite subdirectly irreducible unary algebra through its monolith.

Proof. Put $n=|A|$ and $w=f_{1} \ldots f_{n}$. For every $c \in A$ there is a unique number $i \in\{0, \ldots, n-1\}$ with $c=w_{\langle i\rangle}(a)$; denote this $i$ by $I(c)$. Let $p$ be a prime number greater than $2 n(n+1)$. Denote by $+^{\prime}$ the addition modulo $p$. Put $B=A \times\{0, \ldots, p-1\}$. Define $\sigma$-operations on $B$ by

$$
f(c, i)=\left\{\begin{array}{l}
(f(c), i) \text { if } f=f_{I(c)+1} \\
\left(f(c), i+{ }^{\prime} 1\right) \text { otherwise. }
\end{array}\right.
$$

Evidently, $A$ is a homomorphic image of $B$ under $(c, i) \mapsto c$. We are going to show that the kernel $R$ of this homomorphism is the monolith of $B$. We have $((c, i),(d, j)) \in R$ if and only if $c=d$. Let $\sim$ be a nontrivial congruence of $B$. We need to show that $R$ is contained in $\sim$.

Let $c, d$ be two elements of $A$. By a path from $c$ to $d$ we mean a word $g_{1} \ldots g_{k}$ such that $d=g_{k} \ldots g_{1}(c)$. Such a path is said to be canonical if for every $i=1, \ldots, k, g_{i}=f_{I\left(g_{i-1} \ldots g_{1}(c)\right)+1}$. Now for every $c, d$ there exists a canonical path from $c$ to $d$ of length at most $n$, and also a noncanonical path from $c$ to $d$ of length at most $n$. For $i=0, \ldots, p-1$ we have $g_{k} \ldots g_{1}(c, i)=\left(d, i+{ }^{\prime} j\right)$ where $j=0$ if the path is canonical and $1 \leq j \leq k$ otherwise.

Let us first prove that if $(c, i) \sim(c, j)$ for some $c$ and some $i \neq j$ then $R$ is contained in $\sim$. By taking a canonical path from $c$ to $a$ we get $(a, i) \sim$ 
$(a, j)$. Let $g \in \sigma$ be different from $f_{1}$. We have $g(a, i) \sim g(a, j)$, i.e., $\left(g(a), i++^{\prime} 1\right) \sim\left(g(a), j+^{\prime} 1\right)$. Applying a canonical path from $g(a)$ to $a$ we get $\left(a, i+{ }^{\prime} 1\right) \sim\left(a, j+^{\prime} 1\right)$. Since $p$ is a prime number, it follows that $(a, k) \sim(a, l)$ for all $k$ and $l$. If $d$ is any element of $A$ then applying a canonical path from $a$ to $d$ we get $(d, k) \sim(d, l)$.

Now let $(c, i) \sim(d, j)$ where $c \neq d$. Without loss of generality, $j=i+^{\prime} l$ where $0 \leq l<\frac{p}{2}$. The canonical path from $c$ to $c$ of length $n$ is a noncanonical path from $d$ to some element $e_{1}$, because $w$ is an irreducible word. Applying this path we get $(c, i) \sim\left(e_{1}, i+{ }^{\prime} l+{ }^{\prime} k_{1}\right)$ for some $e_{1}, k_{1}$ with $1 \leq k_{1} \leq n$. If $e_{1}=c$ then $(c, i) \sim\left(c, i+{ }^{\prime} l+{ }^{\prime} k_{1}\right)$ where $i \neq i+{ }^{\prime} l+{ }^{\prime} k_{1}$ and we are done by above. Otherwise, apply the same path to obtain $(c, i) \sim$ $\left(e_{2}, i+{ }^{\prime} l+{ }^{\prime} k_{1}+{ }^{\prime} k_{2}\right)$ for some $e_{2}, k_{2}$ with $1 \leq k_{2} \leq n$. Continue in this way $n+1$ times. If it did not happen that $e_{m}=c$ for some $m$, in which case we would be finished by above, we get $(c, i) \sim\left(e_{m}, i+{ }^{\prime}\left(l+k_{1}+\cdots+k_{m}\right)\right)$ for all $m=1, \ldots, n+1$. Since $p$ was so large, we have $m \leq l+k_{1}+\cdots+k_{m}<$ $\frac{p}{2}+m n<p$ and since all $k_{m} \neq 0$, the numbers $i+^{\prime}\left(l+k_{1}+\cdots+k_{m}\right)$ are pairwise distinct. However, the elements $e_{1}, \ldots, e_{n+1}$ cannot be pairwise distinct. So, we get into the previous case.

Lemma 4. Let $a, w$ be an irreducible h-pair for a finite unary algebra $A$. Then there exist a finite subdirectly irreducible unary algebra $C$ and a homomorphism $H$ of $C$ onto $A$ such that for every element $c \in A$ there exists an element $c^{\prime} \in C$ with $H\left(c^{\prime}\right)=c$ and $c^{\prime} \neq f(d)$ for all $f \in \sigma$ and all $d \in C$.

Proof. Define $B$ in the same way as in Lemma 3. Put $C=A \times\{0, \ldots, p\}$ and define operations on $C$ in such a way that $B$ is a subalgebra of $C$ and $f(c, p)=(f(c), I(c))$ (we have $I(c)<n<p)$. Clearly, $A$ is a homomorphic image of $C$ under $(c, i) \mapsto c$. Define a congruence $S$ of $C$ by $((c, i),(d, j)) \in S$ if and only if either $(c, i)=(d, j)$ or $c=d$ and $i, j<p$. In order to prove that $S$ is the monolith of $C$, it is sufficient (by the proof of Lemma 3 ) to show that if $(c, i) \sim(d, p)$ where $(c, i) \neq(d, p)$ then $\left(c^{\prime}, j\right) \sim\left(d^{\prime}, k\right)$ for some $\left(c^{\prime}, j\right) \neq\left(d^{\prime}, k\right)$ with $j, k<p$. If $i=p$ then $c \neq d, I(c) \neq I(d)$ and so it is sufficient to apply any operation to $(c, p) \sim(d, p)$. Let $i \neq p$. If $i \neq I(d)$, apply $f_{I(c)+1}$; and if $i=I(d)$, apply any operation different from $f_{I(c)+1}$.

Lemma 5. Let $a, w$ be an irreducible h-pair for a finite unary algebra $A$ and let $N$ be a positive integer. Then there exist a finite subdirectly irreducible unary algebra $C$ and a homomorphism $H$ of $C$ onto $A$ such that for every element $c \in A$ there exist at least $N$ distinct elements $c^{\prime} \in C$ with $H\left(c^{\prime}\right)=c$ and $c^{\prime} \neq f(d)$ for all $f \in \sigma$ and all $d \in C$.

Proof. $a, w^{N}$ is an e-pair for $A$. With respect to this e-pair, apply Lemma 1 and Lemma 2 to obtain an algebra $A^{\prime}$ without proper subalgebras and a homomorphism of $A^{\prime}$ onto $A$ such that for every element $c \in A$ there are $N$ elements of $A^{\prime}$ mapped onto $c$. Then apply Lemma 4 to this algebra $A^{\prime}$.

Lemma 6. Let $A$ be a finite unary algebra such that one of the following two cases takes place: 
(1) A has a smallest subalgebra $U$;

(2) A has two disjoint subalgebras $U,\{a\}$ such that the partial algebra $A-\{a\}$ has a smallest subalgebra $U$.

Then $A$ is a homomorphic image of a finite subdirectly irreducible algebra.

Proof. In both cases put $A_{0}=U$, so that $A_{0}$ has no proper subalgebras. By Lemma 5 there exist, for every positive integer $N$, a subdirectly irreducible algebra $B_{0}$ and a homomorphism $H_{0}$ of $B_{0}$ onto $A_{0}$ such that for every element $c \in A_{0}$ there are at least $N$ distinct elements $c^{\prime} \in B_{0}$ with $H_{0}\left(c^{\prime}\right)=c$ and $c^{\prime} \neq f(d)$ for all $f \in \sigma$ and all $d \in B_{0}$.

Define subsets $A_{0}, A_{1}, \ldots$ of $A$ by induction as follows: $A_{i+1}$ is the set of all elements $b \in A$ not belonging to $A_{0} \cup \cdots \cup A_{i}$ such that $f(b) \in A_{i}$ for some $f \in \sigma$. Let $K$ be the largest integer with $A_{K}$ nonempty. We have $A=A_{0} \cup \cdots \cup A_{K}$ in case (1) and $A=A_{0} \cup \cdots \cup A_{K} \cup\{a\}$ in case (2). If $K=0$, we can use Lemma 3 ; assume $K>0$.

Put $B_{K}=A_{K}$ and let $H_{K}$ be the identity on $B_{K}$. For every $i=K-$ $1, \ldots, 1$ define a set $B_{i}$ and a mapping $H_{i}$ of $B_{i}$ into $A_{i}$ as follows: $B_{i}$ is the disjoint union of $A_{i}$ with the set of ordered pairs $(b, f)$ such that $b \in B_{i+1}, f \in \sigma$ and $f\left(H_{i+1}(b)\right) \in A_{i}$; put $H_{i}(b, f)=f\left(H_{i+1}(b)\right)$, and let the restriction of $H_{i}$ to $A_{i}$ be the identity. Take $N$ so large that for every $f \in \sigma$ there exists an injective mapping $G_{f}$ of $B_{1}$ into $B_{0}$ with this property: if $b \in B_{1}$ and $f\left(H_{1}(b)\right) \in A_{0}$ then $H_{0} G_{f}(b)=f\left(H_{1}(b)\right)$ and $G_{f}(b)$ is not in the range of any fundamental operation of $B_{0}$. Put $B^{*}=B_{K} \cup \cdots \cup B_{1} \cup B_{0}$ and $H^{*}=H_{K} \cup \cdots \cup H_{1} \cup H_{0}$; in case (1) put $B=B^{*}$ and $H=H^{*}$, while in case (2) put $B=B^{*} \cup\{a\}$ and let $H$ be the extension of $H^{*}$ by $H(a)=a$. We are going to define operations on $B$. In case (2) put $f(a)=a$ for all $f \in \sigma$. Let $f \in \sigma$ and $b \in B_{i}$. If $i=0$, define $f(b)$ in $B$ in the same way as in $B_{0}$ (so that $B_{0}$ is a subalgebra of $B$ ). If $i \geq 2$ and $f(H(b)) \in A_{i-1}$, put $f(b)=(b, f)$ (this is an element of $B_{i-1}$ ). If $i \geq 2$ and $f(H(b)) \notin A_{i-1}$ (so that either $f(H(b)) \in A_{j}$ for some $j \geq i$ or $(2)$ takes place and $f(H(b))=a$ ), put $f(b)=f(H(b))$. If $i=1$ and $f(H(b)) \in A_{0}$, put $f(b)=G_{f}(b)$. If $i=1$ and $f(H(b)) \notin A_{0}$, put $f(b)=f(H(b))$.

One can easily check that $H$ is a homomorphism of $B$ onto $A$. We are going to prove that the union $R$ of the monolith of $B_{0}$ with $\operatorname{id}_{B}$ is the monolith of $B$. Let $b, c$ be two distinct elements of $B$ and $\sim$ be a congruence with $b \sim c$. It is sufficient to prove that there exist two distinct $\sim$-related elements in $B_{0}$.

Consider first the case $c=a$. We have $b \sim a$, so that $f(b) \sim f(a)=a$ for all $f \in \sigma$. Since $g_{k} \ldots g_{1}(b) \in B_{0}$ for some $g_{1}, \ldots, g_{k} \in \sigma$, we get $d \sim a$ for some $d \in B_{0}$. Then also $f(d) \sim a$ for all $f \in \sigma$, so that $d \sim f(d)$ where both $d$ and $f(d)$ belong to $B_{0}$; for at least one $f, d \neq f(d)$.

It remains to consider the case when $b \in B_{i}$ and $c \in B_{j}$ for some $i, j$. Without loss of generality, $i \leq j$. If $i=j=0$, we are done. 
If $i=0$ and $j=1$ then there exists an $f \in \sigma$ with $f(c) \in B_{0}$; we have $f(b) \sim f(c)$ and these two elements are distinct, since $f(c)$ is not in the range of $f$ restricted to $B_{0}$.

If $i=0$ and $j \geq 2$ then there exists an $f \in \sigma$ with $f(c) \in B_{j-1}$, so $j$ can be reduced by 1 and (after several such steps) this takes us into the previous case.

If $i=j=1$ then there are $f, g \in \sigma$ with $f(b) \in B_{0}$ and $g(c) \in B_{0}$; if $f(c) \in B_{0}$, take $g=f$. If $f=g$ then applying $f$ to $b \sim c$ yields a pair of $\sim-$ related elements in $B_{0}$; these two elements are distinct, since $G_{f}$ is injective. If $f \neq g$ then applying $f$ to $b \sim c$ yields a pair of $\sim$-related elements, one of which does while the other does not belong to $B_{0}$ and the previous case applies.

If $i=1$ and $j \geq 2$, then an application of a suitable $f$ to $b \sim c$ yields a pair of $\sim$-related elements, one of which does while the other does not belong to $B_{0}$.

Finally, if $i, j \geq 2$ then take an $f \in \sigma$ with $f(b) \in B_{i-1}$. We have $f(b) \sim f(c)$ where $f(b) \in B_{i-1}, f(c) \in B_{k}$ for some $k \geq 1$ (or $f(c)=a$ ) and $f(b) \neq f(c)$.

Theorem 7. The following are equivalent for a finite unary algebra A:

(1) $A$ is a homomorphic image of a subdirectly irreducible algebra;

(2) $A$ is a homomorphic image of a finite subdirectly irreducible algebra;

(3) Either $A$ has a smallest subalgebra or A has two disjoint subalgebras $U,\{a\}$ such that the partial algebra $A-\{a\}$ has a smallest subalgebra $U$.

(4) A has a nonempty intersection of the set of all subalgebras $B$ of $A$ such that $|B| \geq 2$.

Proof. (3) implies (2) by Lemma 6, (2) implies (1) clearly. Next we prove that (1) implies (4). Let $h$ be a homomorphism of a subdirectly irreducible algebra $B$ onto $A$. Suppose that $A$ has a subalgebra $U,|U| \geq 2$. Then $U^{\prime}=h^{-1}(U)$ is a subalgebra of $B,\left|U^{\prime}\right| \geq 2$ and $U^{\prime 2} \cup \mathrm{id}_{B}$ is a nontrivial congruence of $B$, denoted by $\Theta_{U^{\prime}}$. If all such subalgebras $U$ of $A$ with at least two elements had empty intersection, then the corresponding inverse images $U^{\prime}$ would have empty intersection. However, their corresponding congruences $\Theta_{U^{\prime}}$ would all be nontrivial, and would have trivial intersection, which contradicts the assumption that $B$ is subdirectly irreducible.

Assume now (4) and denote $V$ the intersection of all subalgebras of $A$ with at least two elements. Assume that the intersection of all subalgebras of $A$ is empty. It means that the intersection of $V$ and all one-element subalgebras of $A$ is empty. Since $A$ cannot have three pairwise disjoint subalgebras (by (4)), it has at most two one-element subalgebras, at most one in $V$ and exactly one outside of $V$ - denote it $\{a\}$. Hence the intersection of all subalgebras of $V$ is non-empty (it is either $V$ itself or the one-element subalgebra of $V$ ) and so is that of $A-\{a\}$. 


\section{REFERENCES}

[1] S. Bulman-Fleming, E. Hotzel and J. Wang, Semigroups that are factors of subdirectly irreducible semigroups by their monolith. Algebra Universalis 51 (2004), 1-7.

[2] J. Ježek and T. Kepka, The factor of a subdirectly irreducible algebra through its monolith. Algebra Universalis 47 (2002), 319-327.

[3] T. Kepka, On a class of subdirectly irreducible groupoids. Acta Univ. Carolinae Math. Phys. 22/1 (1981), 17-24.

[4] T. Kepka, A note on subdirectly irreducible groupoids. Acta Univ. Carolinae Math. Phys. 22/1 (1981), 25-28.

[5] T. Kepka, On a class of groupoids. Acta Univ. Carolinae Math. Phys. 22/1 (1981), $29-49$.

[6] R. McKenzie, G. McNulty and W. Taylor, Algebras, Lattices, Varieties, Volume I. Wadsworth \& Brooks/Cole, Monterey, CA, 1987.

[7] R. McKenzie, D. Stanovský, Every quasigroup is isomorphic to a subdirectly irreducible quasigroup modulo its monolith. Submitted.

[8] D. Stanovský, Homomorphic images of subdirectly irreducible groupoids. Comment. Math. Univ. Carolinae 42/3 (2001), 443-450.

[9] M. Yoeli, Subdirectly irreducible unary algebras. Am. Math. Mon. 74 (1967), 957-960.

Department of Algebra, Charles University, Sokolovská 83, 18675 Praha 8, Czech Republic

E-mail address: jezek@karlin.mff.cuni.cz

E-mail address: stanovsk@karlin.mff.cuni.cz

Department of Mathematics and Informatics, Faculty of Science, University of Novi Sad, trg Dositeja Obradovića 3, 21000 Novi Sad, Serbia and MonTENEGRO

E-mail address: pera@im.ns.ac.yu 